\title{
Accelerated Gradient Based Optimization Using Adjoint Sensitivities
}

\author{
Natalia K. Nikolova, Member, IEEE, Reza Safian, Ezzeldin A. Soliman, Associate Member, IEEE, \\ Mohamed H. Bakr, Member, IEEE, and John W. Bandler, Fellow, IEEE
}

\begin{abstract}
An electromagnetic feasible adjoint sensitivity technique (EM-FAST) has been proposed recently for use with frequency-domain solvers . It makes the implementation of the adjoint variable approach to design sensitivity analysis straightforward while preserving the accuracy at a level comparable to that of the exact sensitivities. The overhead computations associated with the estimation of the sensitivities in addition to the system analysis are due largely to the calculation of the derivatives of the system matrix. Here, we describe the integration of the EM-FAST with two methods for accelerated estimation of these derivatives: the boundary-layer concept and the Broyden update. We show that the Broyden update approach (Broyden-FAST) leads to an algorithm whose efficiency is problem independent and allows the computation of the response and its gradient through a single system analysis with practically no overhead. Both approaches are illustrated through the design of simple antennas using method of moments solvers.
\end{abstract}

Index Terms-Adjoint sensitivities, antenna design, Broyden update, design methodology, method of moments (MoM), optimization, sensitivity.

\section{INTRODUCTION}

$\mathbf{T}$ RADITIONAL full-wave electromagnetic (EM) solvers do not compute the gradient of the response (e.g., $S$-parameters, input impedance or antenna gain) with respect to the design parameters, which relate to the geometry and the materials of the structure. Commercial high-frequency CAD software typically resorts to finite-difference approximations of the response sensitivities, which are numerically inefficient but simple to implement with existing EM solvers. To compute the response and its sensitivities, such an approach requires a minimum of $n+1$ full-wave analyses, $n$ being the number of the design parameters. This approach is also known as the perturbation approximate sensitivity technique (PAST) [1]. Higher-order approximations may also be used at the expense of an increased number of simulations. They are feasible when

Manuscript received December 12, 2002; revised September 30, 2003. This work was supported in part by the Natural Sciences and Engineering Research Council of Canada under Grants OGP0227660-03, OGP0007239, OGP0249780-02, STR234854-00, through the Micronet Network of Centres of Excellence and Bandler Corporation.

N. K. Nikolova, R. Safian, E. A. Soliman, and M. H. Bakr are with the Department of Electrical and Computer Engineering, McMaster University, Hamilton, ON L8S 4K1, Canada.

J. W. Bandler is with with the Department of Electrical and Computer Engineering, McMaster University, Hamilton, ON L8S 4K1, Canada and also with Bandler Corporation, Dundas, ON L9H 5E7, Canada.

Digital Object Identifier 10.1109/TAP.2004.832313 sufficient database for the system response in the design parameter space becomes available. Such response data, for example, would gradually accumulate during optimization.

It is possible to derive exact sensitivity expressions for the state variables of a system by directly differentiating its equations with respect to the desired design parameters. For example, in [2], a mixed potential integral equation is developed for the current density derivatives with the method of moments (MoM) applied to planar multilayer structures. This equation, when solved together with the original electric field integral equation, yields both the currents and their derivatives with respect to the design parameters. Such an approach—generally referred to as sensitivity analysis via direct differentiation - can be applied to both steady-state [3], [4] and dynamic [5] systems. For each design parameter, an additional linear system analysis is required to obtain the respective response derivative. Each of these analyses is characterized by the same system matrix, which is also identical with the original system matrix.

A more efficient design sensitivity analysis is provided by the adjoint variable method [3]-[7]. It reduces the computational overhead of the sensitivity computation to just one additional linear system analysis where the system matrix is the transpose of that of the original problem. Thus, its computational overhead is $n$ times smaller than that of the direct differentiation approach and is practically independent of the number of design parameters $n$. Adjoint-based design sensitivity analysis of microwave structures has been first formulated in terms of circuit concepts rather than field concepts, and it is referred to as the adjoint network method [7]-[12].

To obtain exact sensitivities, both the direct differentiation and the adjoint-variable techniques require the analytical derivatives of the system matrix with respect to the design parameters. This constitutes a major difficulty in applications with full-wave EM solvers for research or commercial design software. Recently, adjoint variable approaches were used with the finite-element method (FEM); see, for example, [13]-[15]. The FEM is well suited for exact sensitivity calculations because of the analytical relation between the coefficients of the FEM matrix and the coordinates of the vertices of the finite element grid. This analytical relation, however, is not trivial. Its implementation in the computation of the derivatives of the FEM system matrix with respect to any geometrical or material design parameter is in practice difficult and, to our knowledge, has not been exploited yet in commercial high-frequency CAD software.

A similar difficulty exists with the exact sensitivities for the MoM. The different varieties of MoM techniques rely on specific Green's functions, as well as different basis and weighting 
functions. The dependence of the system matrix coefficients on possible geometry perturbations is involved and case specific [2], [16].

In summary, exact sensitivities appear to be often impractical in full-wave EM analysis for two reasons: (1) the analytical preprocessing is involved and solver specific; (2) the implementation requires thorough reworking of the analysis engine. The second requirement is especially unattractive in the development of commercial software.

Recently, a feasible adjoint-sensitivity technique (FAST) for applications with full-wave EM solvers (EM-FAST) has been proposed [17]. It uses finite differences to approximate the derivatives of the system matrix. Its implementation in a versatile $\mathrm{CAD}$ environment is straightforward since it requires minor additions to existing frequency-domain computational algorithms. Its accuracy is comparable to that of the analytical exact sensitivities. Its overhead is mostly due to the finite-difference computation of the derivatives of the system matrix, and it is equivalent to that of the exact sensitivity calculations. Here, we propose the use of two techniques-the boundary-layer concept and the Broyden update- to enhance the speed of the EM-FAST, which is crucial in gradient-based optimization. There is a certain loss of accuracy; however, the approximated sensitivities are sufficiently accurate to efficiently guide the optimization toward the optimal design. In applications requiring higher accuracy of the response gradient such as tolerance or yield analysis, the original EM-FAST may be preferable.

We start with a brief outline of the EM-FAST [17] and its computational requirements. We then discuss ways to accelerate its performance through the boundary layer concept (BLC) and the Broyden update. The resulting algorithms offer significant CPU time reduction in comparison with the original EM-FAST on the order of the number of design parameters $n$. The savings in comparison with the traditional finite-difference gradient approximation applied directly to the set of responses (e.g., PAST) are drastic, especially in the case of multiple design variables.

\section{Feasible Adjoint Sensitivity Analysis}

\section{A. Definitions and Notations in Adjoint Sensitivity Analysis}

Consider the system of complex-valued equations arising form the discretization of a linear EM problem

$$
Z(x) I=V
$$

where, $\boldsymbol{x}=\left[x_{1} \ldots x_{n}\right]^{T}$ is the vector of design parameters. These parameters typically have real values related to the geometry and the materials of the structure. $I=\left[I_{1} \ldots I_{m}\right]^{T}$ is the state variable vector, e.g., complex-valued current distribution in the MoM; $V$ is the excitation vector; $Z$ is the system matrix whose complex coefficients depend on the geometry and materials.

The objective of sensitivity analysis is to determine the gradient of a properly defined response function $f(x, \bar{I}(x))$ at the current solution $\bar{I}$ of (1) with respect to the design parameters $\boldsymbol{x}$

$$
\nabla_{\boldsymbol{x}} f, \quad \text { subject to } \boldsymbol{Z}(\boldsymbol{x}) \boldsymbol{I}=\boldsymbol{V} \text {. }
$$

We assume that the response is a scalar function, which is differentiable in $\boldsymbol{x}$ and $\boldsymbol{I}$. We define the gradient operator as a row operator

$$
\nabla_{\boldsymbol{x}} f=\left[\begin{array}{llll}
\frac{\partial f}{\partial x_{1}} & \frac{\partial f}{\partial x_{2}} & \cdots & \frac{\partial f}{\partial x_{n}}
\end{array}\right]
$$

When the gradient operator acts on a vector, e.g., $V$, the result is a matrix

$$
\nabla_{\boldsymbol{x}} \boldsymbol{V}=\left[\begin{array}{ccc}
\frac{\partial V_{1}}{\partial x_{1}} & \cdots & \frac{\partial V_{1}}{\partial x_{n}} \\
\vdots & & \vdots \\
\frac{\partial V_{m}}{\partial x_{1}} & \cdots & \frac{\partial V_{m}}{\partial x_{n}}
\end{array}\right]
$$

The optimization problem is formulated as

$$
\boldsymbol{x}^{*}=\arg \left\{\min _{\boldsymbol{x}} f(\boldsymbol{x}, \boldsymbol{I}(\boldsymbol{x}))\right\}
$$

where $f$ is the objective function to be minimized, and $\boldsymbol{x}^{*}$ is the vector of optimal design parameters. Gradient-based optimizers require both the response of the current design $f$ and its sensitivity (3) in order to predict the next design iterate.

The sensitivities of the objective function are obtained as [18]

$$
\nabla_{\boldsymbol{x}} f=\nabla_{\boldsymbol{x}}^{e} f+\Re\left\{\hat{\boldsymbol{I}}^{T} \cdot\left[\nabla_{\boldsymbol{x}} \boldsymbol{V}-\nabla_{\boldsymbol{x}}(Z \overline{\boldsymbol{I}})\right]\right\}
$$

where $\hat{\boldsymbol{I}}$ is the solution of the complex adjoint problem

$$
Z^{T} \hat{\boldsymbol{I}}=\hat{\boldsymbol{V}}
$$

in which the adjoint excitation $\hat{V}$ is defined by

$$
\hat{V}=\left[\begin{array}{c}
\frac{\partial f}{\partial \Re I_{1}}-j \frac{\partial f}{\partial \Im I_{1}} \\
\vdots \\
\frac{\partial f}{\partial \Re I_{m}}-j \frac{\partial f}{\partial \Im I_{m}}
\end{array}\right] .
$$

Here, $\Re$ and $\Im$ denote the real and imaginary parts, respectively, of a complex variable. The gradient $\nabla_{x}^{e} f$ reflects the explicit dependence of $f$ on $\boldsymbol{x}$. The matrix $\nabla_{\boldsymbol{x}} \boldsymbol{V}$ would typically be analytically available. In fact, the excitation is often insensitive to changes in the design parameters, i.e., $\nabla_{\boldsymbol{x}} \boldsymbol{V}=\mathbf{0}$. For example, in a microstrip circuit, the excitation is defined at ports located at feed lines. If the design parameter variations affect neither the dielectric constant nor the height of the substrate, nor the width of the feed line, the excitation remains unchanged.

In $\nabla_{\boldsymbol{x}}(Z \bar{I}), \overline{\boldsymbol{I}}$ is a constant vector representing the solution at the current design, i.e., (6) can be written explicitly as

$$
\frac{\partial f}{\partial x_{i}}=\frac{\partial^{e} f}{\partial x_{i}}+\Re\left[\hat{\boldsymbol{I}}^{T} \cdot\left(\frac{\partial \boldsymbol{V}}{\partial x_{i}}-\frac{\partial \boldsymbol{Z}}{\partial x_{i}} \cdot \overline{\boldsymbol{I}}\right)\right], \quad i=1, \ldots, n .
$$

The sensitivity expression (6) is a generalization of the wellknown, linear, real-system sensitivity formula [3], [17].

As evident from (6) and (7), the adjoint approach provides the gradient of the response with respect to all $n$ design parameters with just one additional system analysis (7) whose system matrix is simply related to that of the original problem (1). When $L U$ factorization of $\boldsymbol{Z}$ is used to solve (1), the $L U$ factors of $\boldsymbol{Z}^{T}$ 
are easily obtained by rearranging the $L U$ factors of $Z$. Thus, the additional system analysis (7) is practically avoided, the overhead being due only to the forward-backward substitutions. In the case of iterative solvers - often used when $\boldsymbol{Z}$ is large and/or sparse-a complete additional system analysis seems imperative.

\section{B. The Feasible Technique}

The matrices $\partial Z / \partial x_{i}, i=1, \ldots, n$, which we refer to as derivative matrices, may be analytically available, as is the case with the FEM. Then the sensitivities obtained with (9) are exact. The calculation of an analytically available $\partial \boldsymbol{Z} / \partial x_{i}$ matrix at the current design is computationally equivalent to a $Z$-matrix fill; therefore, at each design iteration, the equivalent of $n+1$ matrix fills is needed. Thus, the advantage of analytically available derivative matrices is in the accuracy of the derivative estimation rather than in its computational efficiency. When the $L U$ factors of the system matrix are available from the analysis of (1), the computation of the derivative matrices determines the overhead associated with the sensitivity analysis since it is far more computationally demanding than the forward-backward substitutions when solving the adjoint problem (7). When the system equations of (1) and (7) are solved iteratively, the additional (adjoint) system analysis determines the computational overhead. Even in this case, the reduction of the time to estimate the derivative matrices is desirable.

In full-wave EM analysis usually the derivative matrices $\partial Z / \partial x_{i}, i=1, \ldots, n$, are not analytically available or they are too complicated to obtain for the purposes of general and versatile design software. Then, we can resort to the finite-difference approximation $\Delta Z / \Delta x_{i}, i=1, \ldots, n$ [17], which requires $n$ additional $Z$-matrix fills if forward (or backward) finite differences are used. The associated computational overhead is equivalent to that of the exact sensitivity estimation discussed above. The important advantage here is that the implementation with existing software is simple. The technique does not require any analytical preprocessing, which often restricts the versatility of the algorithm.

We have investigated the accuracy of the sensitivity estimation with the feasible adjoint technique [17] and we have found that it is excellent for relative perturbations $\Delta x_{i} / x_{i}$ between $0.5 \%$ and $2 \%$. The relative error in comparison with the exact sensitivities is well below $1 \%$ for a broad range of values of the design parameters, close to or far from the nulls of the sensitivity curves.

A detailed comparison between the computational requirements of the EM-FAST and the commonly used finite differences applied directly to the response is made in [17]. Here, we only note that the EM-FAST reduces the number of required full-wave analyses by a factor of $n+1, n$ being the number of design parameters. However, there are overhead computations associated with the $n$ additional matrix fills in order to compute $\Delta Z / \Delta x_{i}, i=1, \ldots, n$. In certain cases, e.g., electrically small problems, the MoM matrix fill may account for a significant portion of the CPU time required by the overall analysis (matrix fill plus linear system solution). Such an overhead should not be overlooked in a sequence of repetitive analyses performed during optimization.

\section{ACCELERATED OPTIMIZATION With APPROXIMATED ADJOINT SENSITIVITIES}

There are two techniques which can lead to faster calculation of the derivative matrices. The first one is the boundary-layer concept (BLC) first proposed by Amari [19] in the sensitivity analysis with the direct differentiation method. The acceleration offered by the BLC depends on the relation between the respective design parameter and the geometry of the structure as we explain below. Its computational requirements are dependent on the number of design parameters $n$. It requires modifications of existing EM analysis software, which relate to meshing and matrix building subroutines. Its advantage is that it yields sensitivity estimates of very good accuracy.

The second approach uses Broyden's update to iteratively compute approximate derivative matrices. This approach reduces the overhead drastically since its computational requirements—negligible compared to a matrix fill—practically do not depend on $n$. The Broyden-update approach does not require any modifications of the EM analysis algorithms.

\section{A. BLC With the EM-FAST}

The BLC can be applied with solvers which allow nonuniform discretization and/or unstructured grids, e.g., the FEM and the MoM. The idea is to perturb a certain geometrical parameter (the design parameter $x_{i}$ ) of a structure by respective deformations of as few grid elements as possible. This makes most of the $Z$-matrix coefficients insensitive to the perturbation. Consequently, the matrix derivative $\Delta Z / \Delta x_{i}$ is mostly sparse and only few nonzero coefficients need to be calculated. This is in contrast with the conventional EM-FAST where full remeshing is applied to the perturbed structure, which results in a full $\Delta Z / \Delta x_{i}$ matrix.

We present two examples, which illustrate the BLC. Through them, we investigate the accuracy of the modified EM-FAST algorithm which exploits the BLC.

1) A Dipole of Finite Thickness: We analyze the sensitivity of the input impedance $Z_{\text {in }}$ of a dipole with respect to the normalized length of the dipole $L_{n}=L / \lambda$. The dipole is discretized into segments whose normalized length is uniform and equal to $\delta_{n}=L_{n} / m$ [see Fig. 1(a)]. Here, $m$ is the number of segments. In this example, $m=85$. This example is suitable for design sensitivity tests because the input impedance of a dipole is highly sensitive to its length, especially close to resonance. The thickness of the dipole is represented by the radius of its cross-section, which is constant and set to $a=$ $0.005 \lambda$. The derivatives $\partial R_{\text {in }} / \partial L_{n}$ and $\partial X_{\text {in }} / \partial L_{n}$ are calculated, where $R_{\text {in }}=\Re Z_{\text {in }}$ and $X_{\text {in }}=\Im Z_{\text {in }}$. We use the symmetry of the structure and analyze half of it. The analysis algorithm is based on Pocklington's equation, which is discretized using pulse basis functions and a point-matching technique [20]. Magnetic frill excitation is applied.

Fig. 1(b) shows the perturbed geometry corresponding to a change of length $\Delta L^{(k)}$ at the $k$ th design iteration where only the boundary-layer (edge) segments are changed accordingly. The resulting derivative matrix $\Delta Z / \Delta L_{n}$ has only one row and one column of nonzero elements. Fig. 1(c) shows the same parameter perturbation this time realized with the conventional 


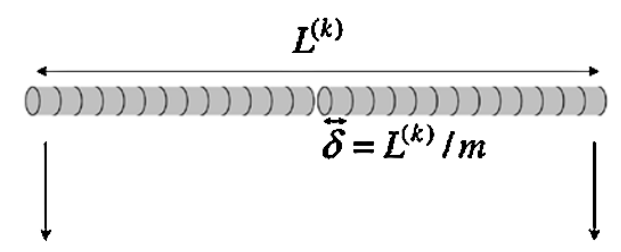

(a)

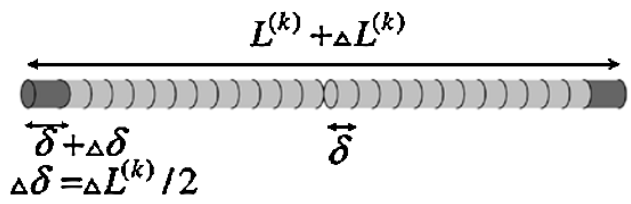

(b)

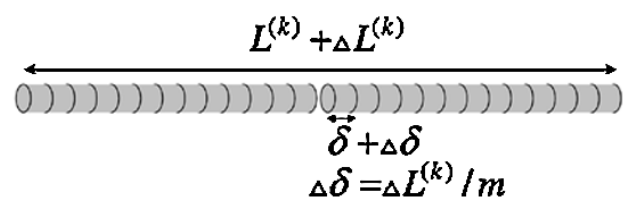

(c)

Fig. 1. Perturbing the length of the dipole at the $k$ th iteration with and without a boundary layer.

EM-FAST approach. Since the centers of all segments in the perturbed structure change their mutual positions, the $\Delta Z / \Delta L_{n}$ matrix is dense.

The input impedance sensitivities are calculated in four different ways. First, the forward finite differences are applied directly to the response $Z_{\text {in }}\left(L_{n}\right)$

$$
\frac{\partial Z_{\text {in }}\left(L_{n}^{(k)}\right)}{\partial L_{n}} \approx \frac{Z_{\text {in }}\left(L_{n}^{(k)}+\Delta L_{n}^{(k)}\right)-Z_{\text {in }}\left(L_{n}^{(k)}\right)}{\Delta L_{n}^{(k)}} .
$$

For each $L_{n}^{(k)}$, the MoM solver is invoked twice to perform the analysis at $L_{n}^{(k)}$, and at $L_{n}^{(k)}+\Delta L_{n}^{(k)}$, where $\Delta L_{n}^{(k)}=0.01 L_{n}^{(k)}$. The sensitivity of $Z_{\text {in }}$ is evaluated in the $L_{n}$ range from 0.3 to 1.2 (see Figs. 2 and 3).

Second, the input impedance sensitivity is computed with the conventional EM-FAST [17]. The derivative matrix $\Delta \boldsymbol{Z} / \Delta L_{n}^{(k)}$ is dense and its coefficients are calculated using forward finite differences applied to each matrix element $Z_{i j}\left(L_{n}\right), i, j=$ $1, \ldots, m$. This requires $m^{2}$ numerical integrations. The increment $\Delta L_{n}^{(k)}$ is again set at $\Delta L_{n}^{(k)}=0.01 L_{n}^{(k)}$. The derivative matrix is then used in (9) to compute $\partial R_{\text {in }} / \partial L_{n}$ and $\partial X_{\text {in }} / \partial L_{n}$. The resulting sensitivity curves are used as a reference as they are the closest to the exact sensitivities [17].

The third and the fourth derivative estimations use the adjoint technique with the BLC. The matrix $\Delta Z / \Delta L_{n}^{(k)}$ is very sparse and its computation is fast as it involves only $2 m$ numerical integrations. The perturbations are set so that the length of the edge elements is increased by $\Delta \delta^{(k)}=0.1 \delta^{(k)}\left(\Delta L^{(k)} \simeq\right.$ $\left.0.002 L^{(k)}\right)$ and $\Delta \delta^{(k)}=0.5 \delta^{(k)}\left(\Delta L^{(k)} \simeq 0.01 L^{(k)}\right)$ for the third and the fourth analysis, respectively. Some accuracy is sacrificed as is clear from Figs. 2 and 3; however, it is sufficient for the purposes of gradient-based optimization. The slight deterioration in accuracy is due to the nonuniformity of the segment

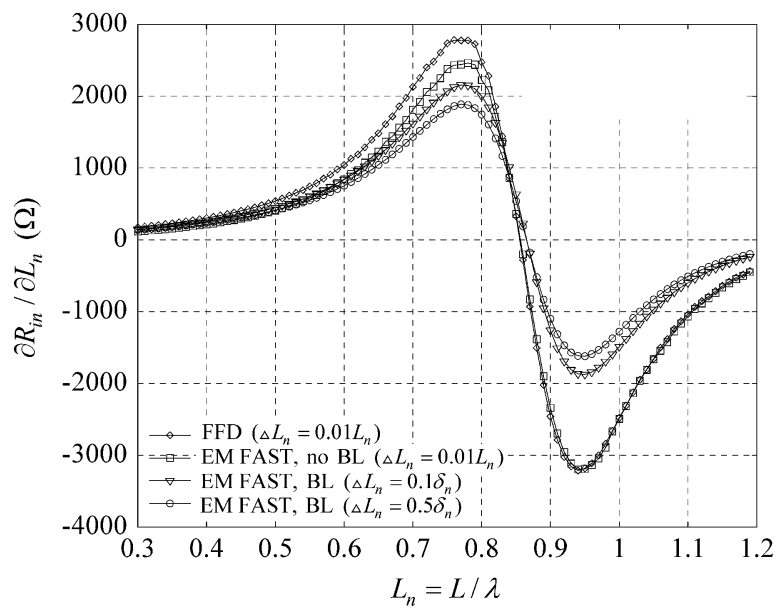

Fig. 2. Derivative of the input resistance of the dipole with respect to its normalized length.

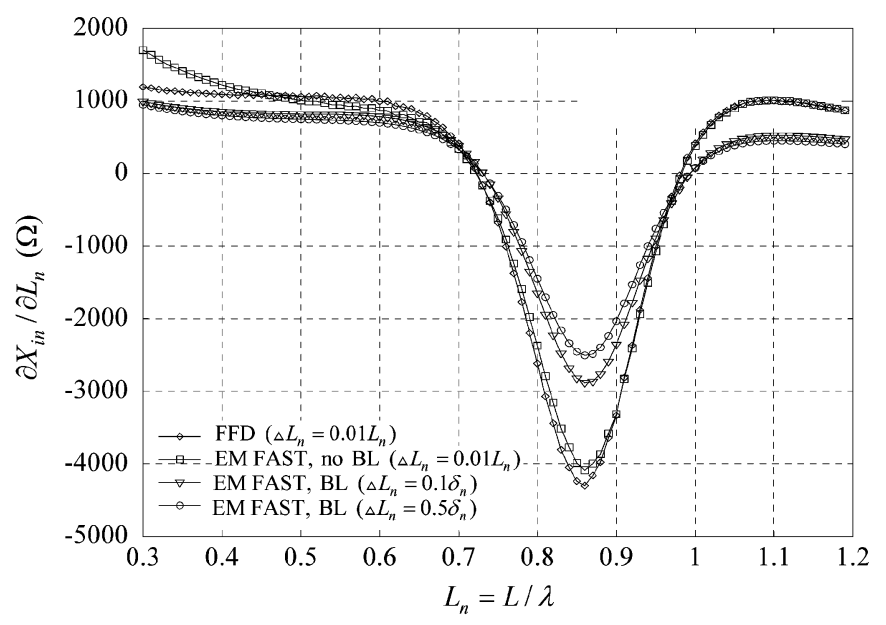

Fig. 3. Derivative of the input reactance of the dipole with respect to its normalized length.

size introduced by the edge-only perturbation. We expect such deterioration to be less when higher-order basis and test functions are used such as triangular functions for wire antennas or rooftops for planar structures.

We now proceed with the optimization of the dipole for an input impedance of $\bar{Z}_{\text {in }}=70 \Omega$. The objective function is defined as

$$
f=\frac{\left|Z_{\text {in }}-\bar{Z}\right|}{\bar{Z}} .
$$

We allow two geometrical parameters to vary: the normalized dipole length $L_{n}$ and the normalized dipole diameter $a_{n}=a / \lambda$. The vector of design parameters is thus $x=\left[\begin{array}{ll}L_{n} & a_{n}\end{array}\right]^{T}$. The following constraints are imposed:

$$
0.45<L_{n}<0.5, \quad 0.003<a<0.006
$$

since this problem is known to be nonunique. The BLC is used to compute the matrix derivative $\Delta Z / \Delta L_{n}$. Notice, however, that it cannot be exploited in the case of the design parameter $a_{n}$ because a change in the antenna diameter affects all $Z$-matrix coefficients. The $\Delta Z / \Delta a_{n}$ matrix is computed with the conventional EM-FAST technique, which requires a full matrix fill. 
TABLE I

OPTIMIZATION OF THE INPUT IMPEDANCE OF THE DIPOLE

\begin{tabular}{cccc}
\hline$k$ & $L_{n}$ & $10^{3} \times a_{n}$ & $f$ \\
\hline 1 & 0.4750 & 4.5 & 0.0406 \\
2 & 0.4688 & 4.9 & 0.0225 \\
3 & 0.4666 & 5.2 & 0.0206 \\
4 & 0.4679 & 5.2 & 0.0069 \\
5 & 0.4685 & 5.1 & 0.0066 \\
6 & 0.4697 & 5.0 & 0.0062 \\
7 & 0.4695 & 4.9 & 0.0041 \\
\hline
\end{tabular}

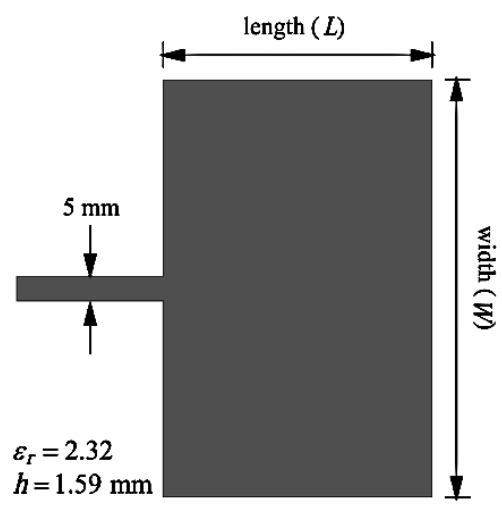

Fig. 4. Geometry of the microstrip-fed patch antenna.

A similar situation would arise in the design of another wire antenna, a Yagi-Uda array. While the BLC is very useful when a design parameter represents the length of a wire, it can offer little or no computational savings if the design parameter is a separation distance between wires. Its efficiency is case specific.

The initial design is $\boldsymbol{x}^{(0)}=\left[\begin{array}{ll}0.45 & 0.004\end{array}\right]^{T}$ where the objective function is $f^{(0)}=0.452$. After seven iterations, an optimal solution is found at $\boldsymbol{x}^{(7)}=\left[\begin{array}{l}0.4695 \\ 0.0049\end{array}\right]^{T}$ with $f^{(7)}=0.0041$, which corresponds to an input impedance of $Z_{\text {in }}=69.71+j 0.02 \Omega$. The progress of the optimization is summarized in Table I. The gradient-based optimization routine of MATLAB ${ }^{1}$ fmincon is used.

2) A Microstrip-Fed Patch Antenna: The EM-FAST is also integrated with an in-house MoM solver, which performs analysis of layered structures. The analysis technique is based on the electric field integral equation. Here, we show an application with the BLC to the optimization of a microstrip-fed rectangular patch antenna. The length $L$ and the width $W$ of the patch are optimized for a maximum real input impedance. The objective function to be minimized is defined as

$$
f(\boldsymbol{x})=-\Re Z_{\text {in }}, \quad \boldsymbol{x}=\left[\begin{array}{ll}
L & W
\end{array}\right]^{T} .
$$

The geometry is shown in Fig. 4. The patch is printed on a substrate of relative dielectric constant $\varepsilon_{r}=2.32$ and height $h=1.59 \mathrm{~mm}$. The initial design is given by $L=55 \mathrm{~mm}$ and $W=82 \mathrm{~mm}$. The operating frequency is set at $2 \mathrm{GHz}$.

\footnotetext{
${ }^{1}$ MATLAB is a registered trademark of The MathWorks, Natick, MA.
}
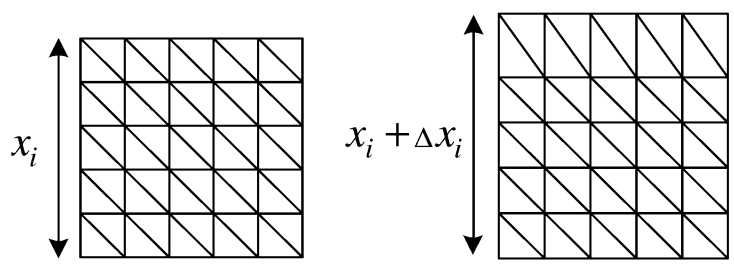

Fig. 5. BLC and the perturbed mesh related to the design parameter $x_{i}$.

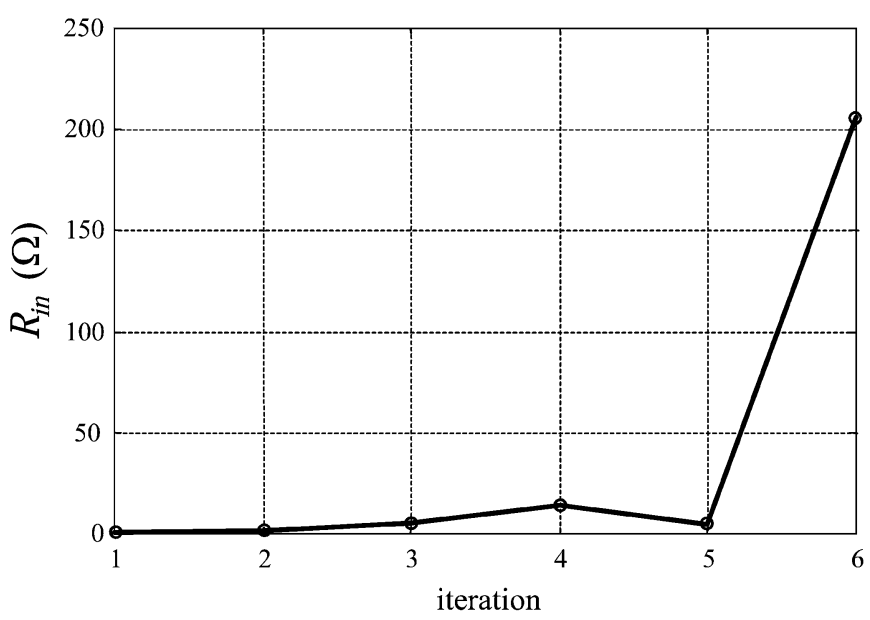

Fig. 6. Progress of $R_{\text {in }}=\Re Z_{\text {in }}$ of the patch antenna during the optimization.

TABLE II

DESIGN PARAMETERS OF THE PATCH AT EACH ITERATION

\begin{tabular}{lllllll}
\hline & $\boldsymbol{x}^{(1)}$ & $\boldsymbol{x}^{(2)}$ & $\boldsymbol{x}^{(3)}$ & $\boldsymbol{x}^{(4)}$ & $\boldsymbol{x}^{(5)}$ & $\boldsymbol{x}^{(6)}$ \\
\hline$L(\mathrm{~mm})$ & 55 & 53.016 & 51.227 & 49.6126 & 51.394 & 50.057 \\
$W(\mathrm{~mm})$ & 82 & 81.741 & 81.542 & 81.4187 & 81.476 & 81.433 \\
\hline
\end{tabular}

The BLC is applied as illustrated in Fig. 5. The calculation of the derivative matrices $\Delta Z^{(k)} / \Delta L$ and $\Delta Z^{(k)} / \Delta W$ is significantly faster than one matrix fill. A matrix fill is equivalent to $\sim\left(N_{L} \times N_{M}\right)^{2}$ integrations, where $N_{L}$ and $N_{M}$ show the number of discrete steps along the length and the width of the patch, respectively. On the other hand, the estimations of $\Delta \boldsymbol{Z}^{(k)} / \Delta L$ and $\Delta \boldsymbol{Z}^{(k)} / \Delta W$ with the BLC are equivalent to $\sim N_{L} \times N_{W}^{2}$ and $\sim N_{L}^{2} \times N_{W}$ numerical integrations, respectively.

The progress of the objective function is shown in Fig. 6 in terms of $R_{\text {in. }}$. The changes of the design parameters with each design iteration are listed in Table II.

\section{B. The Broyden-Update Approach to Matrix Derivative Estimation}

The Broyden update is a classical rank-one formula proposed by Broyden [21] for the approximation of the Jacobian $\nabla_{\boldsymbol{x}} \boldsymbol{F}$ of a vector function $\boldsymbol{F}(\boldsymbol{x})$. If the approximated Jacobian $\nabla_{\boldsymbol{x}} \boldsymbol{F}$ is denoted as $\boldsymbol{G}^{(k)}$ at the $k$ th iteration, Broyden's formula is written as

$$
\begin{aligned}
& \boldsymbol{G}^{(k+1)}=\boldsymbol{G}^{(k)} \\
& \quad+\frac{\boldsymbol{F}\left(x^{(k)}+\boldsymbol{h}^{(k)}\right)-\boldsymbol{F}\left(\boldsymbol{x}^{(k)}\right)-G^{(k)} \boldsymbol{h}^{(k)}}{\boldsymbol{h}^{(k) T} \boldsymbol{h}^{(k)}} \boldsymbol{h}^{(k) T}
\end{aligned}
$$




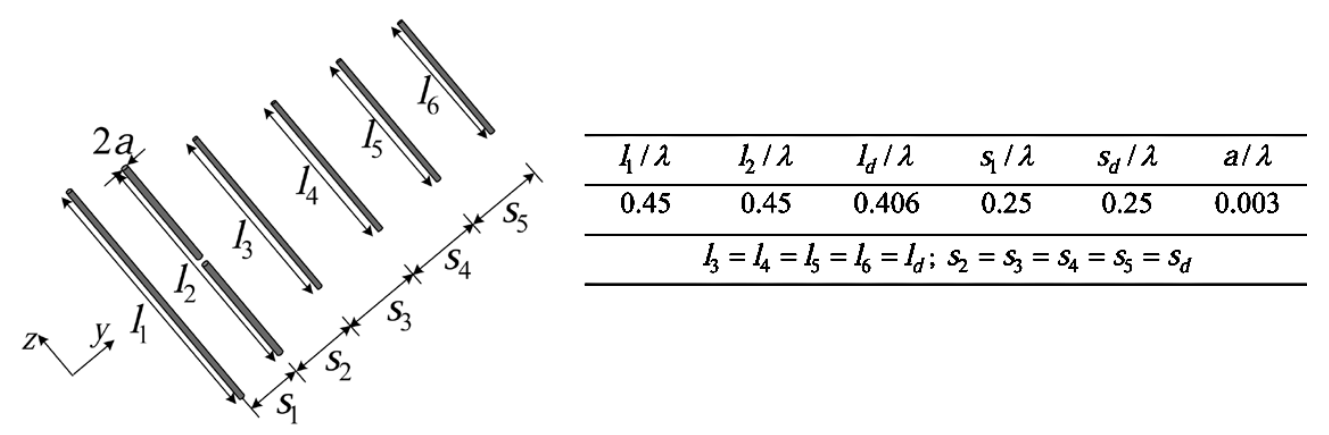

Fig. 7. Geometry of the Yagi-Uda array.

TABLE III

OPTIMIZATION OF THE INPUT IMPEDANCE OF THE YAGI-UdA ARRAY WITH BROYDEN-FAST

\begin{tabular}{cccrrrrc}
\hline$k$ & $l_{1 n}$ & $l_{2 n}$ & $s_{1 n}$ & $s_{2 n}$ & $R_{\text {in }}$ & $X_{\text {in }}$ & $f$ \\
\hline 1 & 0.4500 & 0.4500 & 0.2500 & 0.2500 & 39.0804 & -9.2174 & 0.2858 \\
2 & 0.4594 & 0.4594 & 0.2594 & 0.2594 & 47.9186 & 11.0807 & 0.2255 \\
3 & 0.4544 & 0.4544 & 0.2544 & 0.2544 & 42.8456 & 0.3647 & 0.1433 \\
4 & 0.4555 & 0.4536 & 0.2555 & 0.2555 & 43.2540 & 2.0759 & 0.1412 \\
5 & 0.4534 & 0.4488 & 0.2726 & 0.2534 & 45.0764 & -2.3652 & 0.1092 \\
6 & 0.4534 & 0.4486 & 0.2731 & 0.2534 & 45.1440 & -2.4321 & 0.1086 \\
7 & 0.4534 & 0.4485 & 0.2736 & 0.2534 & 45.2319 & -2.4072 & 0.1068 \\
8 & 0.4567 & 0.4382 & 0.3052 & 0.2567 & 50.7482 & 0.6362 & 0.0196 \\
9 & 0.4560 & 0.4405 & 0.2983 & 0.2560 & 49.5429 & 0.0177 & 0.0091 \\
10 & 0.4562 & 0.4398 & 0.3004 & 0.2562 & 49.9075 & 0.1619 & 0.0037 \\
\hline
\end{tabular}

where $\boldsymbol{h}^{(k)}=\boldsymbol{x}^{(k+1)}-\boldsymbol{x}^{(k)}$ is the increment vector in the design parameter space. It has $n$ elements corresponding to the increment of each design parameter. Broyden's update has been used in a number of applications such as gradient-based optimization where analytical sensitivities are not available [22], the aggressive space mapping technique [23], etc.

We apply Broyden's update to estimate iteratively the derivative matrices $\partial \boldsymbol{Z} / \partial x_{i}, i=1, \ldots, n$, which are subsequently used in the sensitivity expression (9). We refer to this modified adjoint-based technique as Broyden-FAST. In the implementation of (14), every complex-valued matrix coefficient $Z_{i j}(\boldsymbol{x})$ $(i, j=1, \ldots, m)$ is a nonlinear function of the design parameters. We define $\boldsymbol{F}$ as a vector which consists of the real and imaginary parts of all elements of the $Z$ matrix, and $\boldsymbol{G}^{(k)}$ as a matrix which consists of their derivatives. To construct the vector $\boldsymbol{F}$, we stack all the columns of $\Re Z$ in a vector followed by the vector formed by all columns of $\Im \boldsymbol{Z}$. Thus, when $\boldsymbol{Z}$ is an $(m \times m)$ matrix, $\boldsymbol{F}$ is a vector with $2 m^{2}$ elements. A row of the matrix $\boldsymbol{G}^{(k)}$ contains the derivatives of the respective element of the vector $\boldsymbol{F}\left(\boldsymbol{x}^{(k)}\right)$ with respect to all design parameters. Therefore, $\boldsymbol{G}^{(k)}$ is a $\left(2 m^{2} \times n\right)$ matrix.

The approximate derivative matrices generated by the Broyden formula are typically less accurate [22] than those obtained by perturbations in the EM-FAST. Our experience shows that as the optimization proceeds, the response sensitivity estimates produced by Broyden-FAST converge toward the exact sensitivities. As a precaution, in the case of a diverging objective function, the algorithm defaults to the conventional EM-FAST technique.
The advantage of the Broyden update is that it is problem-independent and does not require any modifications of the analysis algorithm. Moreover, its computational requirements are negligible in comparison with the EM-FAST. The response and its gradient are obtained by a single system analysis with practically no overhead regardless of the number of design parameters $n$.

The potential of the Broyden update is demonstrated by two examples: the optimization of a Yagi-Uda array and the optimization of a microstrip-fed patch antenna.

1) Optimization of a Yagi-Uda Array: An initial design of the six-element Yagi-Uda antenna is given in Fig. 7. All dimensions are normalized with respect to the free-space wavelength $\lambda$. We vary the normalized lengths of the reflector and the driven element, $l_{1 n}=l_{1} / \lambda$ and $l_{2 n}=l_{2} / \lambda$, as well as the normalized separation distances $s_{1 n}=s_{1} / \lambda$ and $s_{2 n}=s_{2} / \lambda$. Thus, the vector of design parameters is $\boldsymbol{x}=\left[\begin{array}{lllll}l_{1 n} & l_{2 n} & s_{1 n} & s_{2 n}\end{array}\right]^{T}$. The objective function is set as in (11) with $\bar{Z}=50 \Omega$. The progress of the optimization is summarized in Table III where the changes of the design parameters, the input impedance and the objective function are recorded at each iteration. An optimal solution is reached in nine iterations.

At the $k$ th design iteration, we update the four derivative matrices $\partial \boldsymbol{Z}^{(k)} / \partial x_{i}, i=1, \ldots, 4$, with Broyden's formula and use them to compute the response sensitivities according to (9). The response sensitivities are then used by the optimization algorithm (fmincon) to produce the next design iterate. The Broyden-FAST sensitivities are then compared with the sensitivities calculated off-line where the derivative matrices 


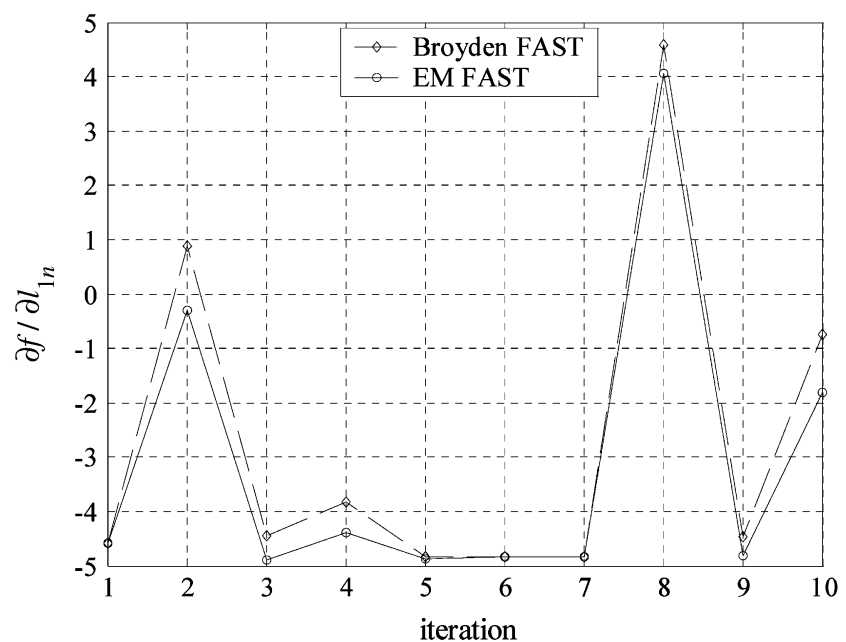

Fig. 8. Sensitivity of the objective function with respect to the length of the reflector during the optimization of $Z_{\text {in }}$ of the Yagi-Uda antenna.

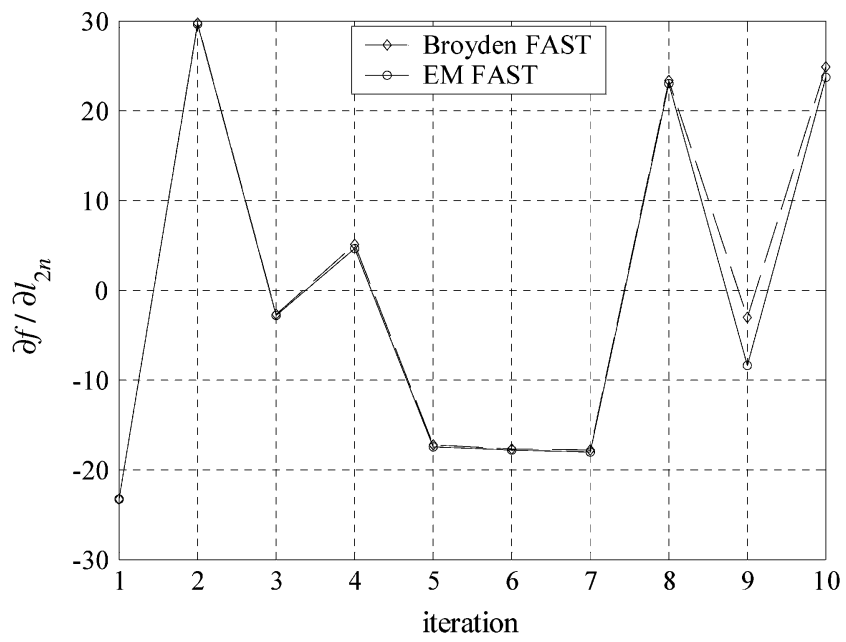

Fig. 9. Sensitivity of the objective function with respect to the length of the driven element $l_{2 n}$ during the optimization of $Z_{\text {in }}$ of the Yagi-Uda antenna.

are obtained by the finite-difference approach of our original technique, the EM-FAST [17]. The sensitivity curves are plotted in Figs. 8-11. At the first iteration only, we compute the derivative matrices using our original approach with forward finite differences and $1 \%$ perturbation over the initial design parameters and assign those to $G^{(1)}$. That is why, at the first iteration, the Broyden-FAST sensitivities and the EM-FAST sensitivities are identical. For all subsequent design iterations, Broyden-FAST uses (14). It is evident that our approach based on the Broyden update produces sufficiently accurate sensitivity results that converge toward the exact sensitivities as the optimization progresses.

To quantify the accuracy of the derivative matrices produced by the Broyden update in the FAST, we compute their global relative errors in $l_{1}$ norm

$$
E\left(x^{(k)}\right)=\frac{\sum_{i, j}\left|\left(\frac{\partial Z_{i j}}{\partial x^{(k)}}\right)_{\text {exact }}-\left(\frac{\partial Z_{i j}}{\partial x^{(k)}}\right)_{\text {Broyden-FAST }}\right|}{\sum_{i, j}\left|\left(\frac{\partial Z_{i j}}{\partial x^{(k)}}\right)_{\text {exact }}\right|},
$$

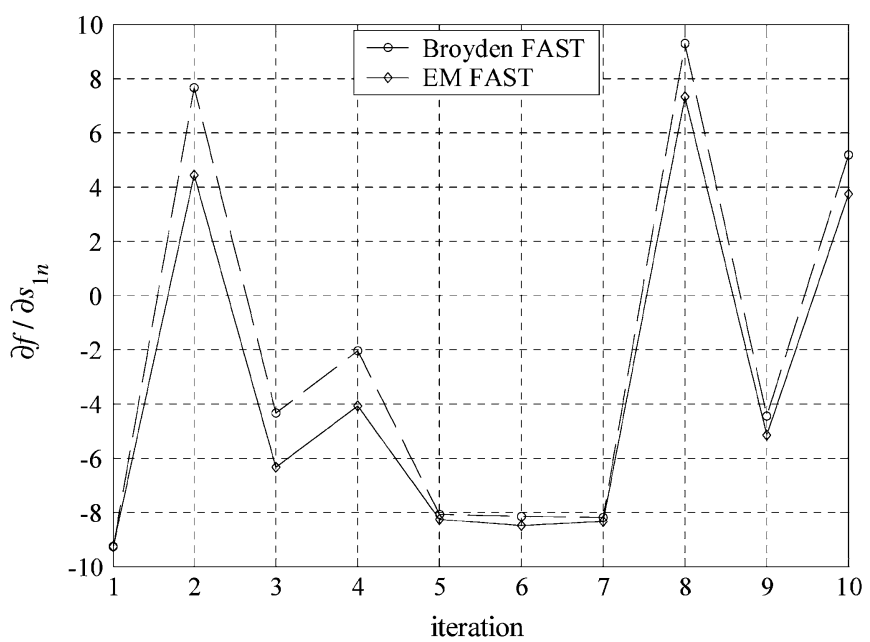

Fig. 10. Sensitivity of the objective function with respect to the separation $s_{1 n}$ during the optimization of $Z_{\text {in }}$ of the Yagi-Uda antenna.

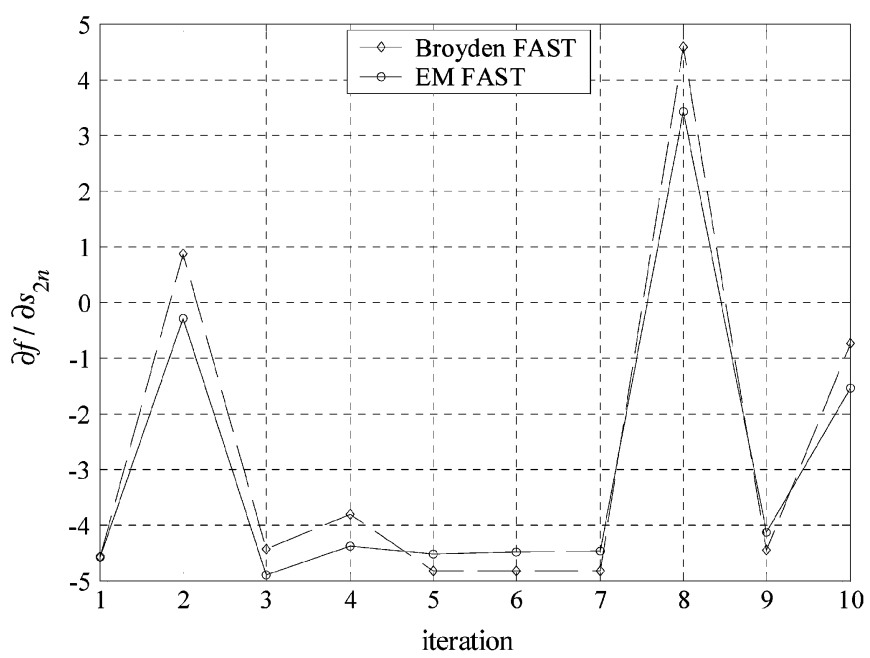

Fig. 11. Sensitivity of the objective function with respect to the separation $s_{2 n}$ during the optimization of $Z_{\text {in }}$ of the Yagi-Uda antenna.

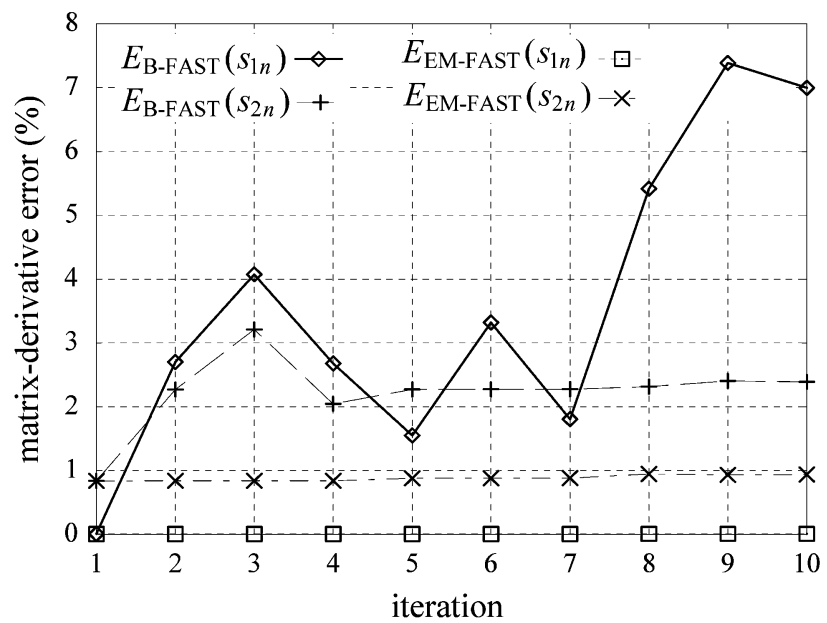

Fig. 12. Global error in the $Z$-matrix derivative estimates of Broyden-FAST and EM-FAST.

Here, the exact derivative is computed using an analytical formula valid for this specific MoM solver [24]. Note that the error estimate (15) operates on complex matrix elements. The errors associated with the normalized separation distances $s_{1 n}$ and $s_{2 n}$ 

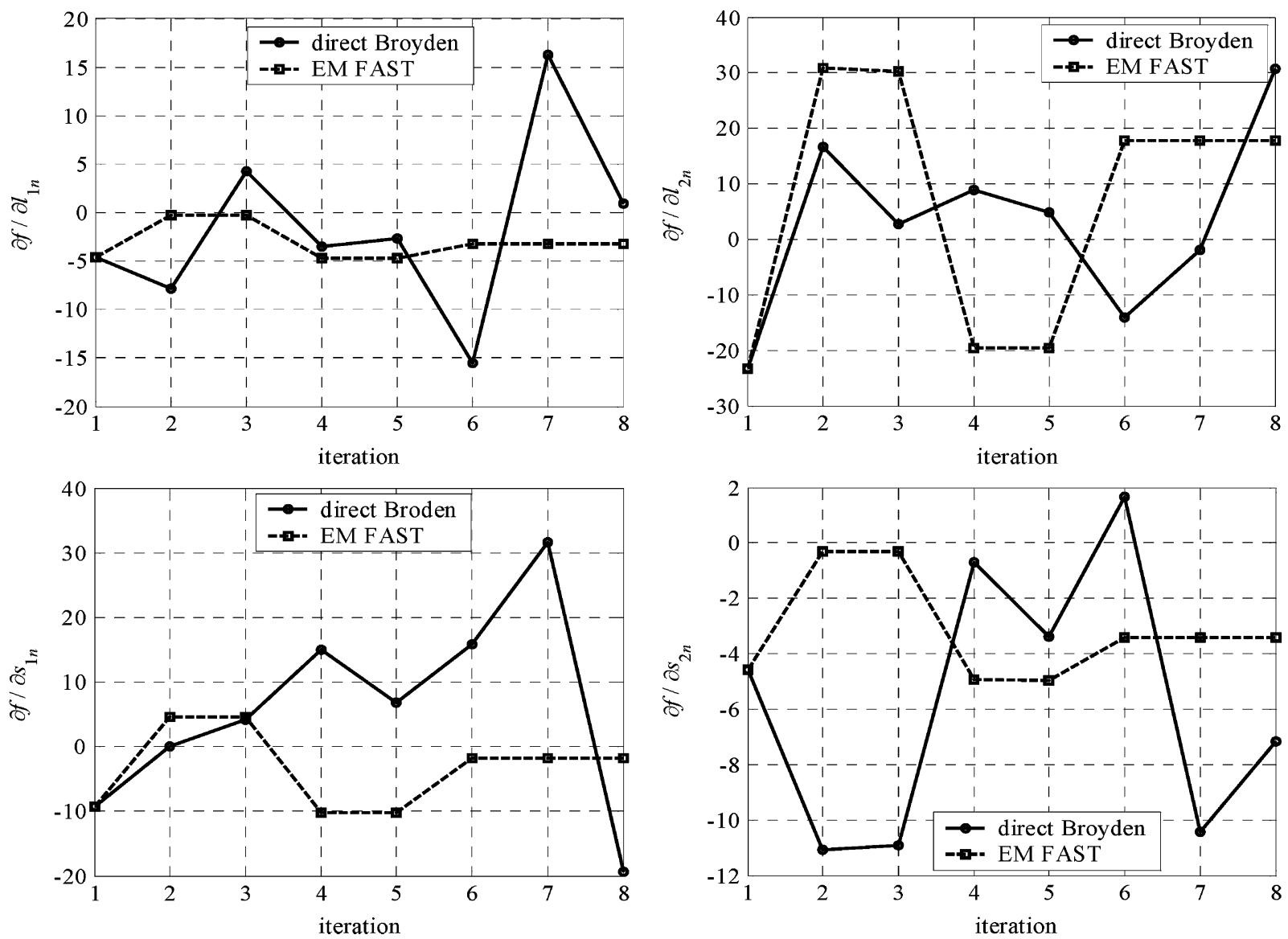

Fig. 13. Sensitivities of the objective function in the optimization of the Yagi-Uda antenna using the direct Broyden update: comparison with reference sensitivities computed with the EM-FAST.

are plotted in Fig. 12. For comparison, we also plot the global errors of the matrix derivatives of the forward finite differencing in the original FAST computed for the same design iterates. As expected, the original FAST is robust while the accuracy of the Broyden estimates may vary throughout the optimization. These variations lead to the small (but observable) differences between the EM-FAST response derivatives and the respective Broyden-FAST estimates (see Figs. 10 and 11). Notice that close to the optimum solution where the optimizer takes very small steps (see Table III), the Broyden update may not perform very well for all design parameters, e.g., $s_{1 n}$, due to the nearly identical $Z$-matrices of the consecutive design iterates; see (14). If necessary, this can be avoided by defaulting to EM-FAST when sufficiently small value of the objective function is achieved. The improved accuracy of the sensitivity estimates may thus improve the convergence of the optimization at its final stages. The hybrid approaches, however, are not a subject of our current discussion.

The Broyden update, of course, can be applied directly to the objective function $f$. However, the objective function usually exhibits strongly nonlinear behavior and sharp sensitivities with respect to the designable parameters. At the same time, the Broyden formula is based on a local linear approximation of the function and thus it performs better with only mildly nonlinear functions. The $\boldsymbol{Z}$-matrix elements, on the other hand, are smooth functions of the shape or material parameters. In fact, the majority of the MoM matrix elements are almost insensitive to shape perturbations except for the diagonal (self-impedance) elements due to the intrinsic dependence on the distance between observation and integration points. Thus, when the Broyden update is applied at the level of the system matrix, better convergence of the sensitivity estimates and of the overall optimization process is expected.

In support of this observation, we repeat the Yagi-Uda antenna design, this time using Broyden's update directly at the level of the objective function in order to estimate its derivatives (direct Broyden approach). We keep the optimization set-up identical to that before: (1) the initial design is as shown in Fig. 7; (2) the objective function is defined as in (11) with $\bar{Z}=50 \Omega$; (3) the same optimization function fmincon of MATLAB is used; (4) the stop criteria $\left|f^{(k+1)}-f^{(k)}\right|<10^{-4}$ and $\left|\boldsymbol{x}^{(k+1)}-\boldsymbol{x}^{(k)}\right|<10^{-6}$ are the same; and (5) the value of the response sensitivity at the first iteration is supplied by the EM-FAST estimate. The direct-Broyden derivatives are used by the optimizer to determine the subsequent design iterates. We also compute the objective function derivatives with the EM-FAST technique off-line in order to supply reference values for comparison.

The direct-Broyden and the reference sensitivity curves are plotted in Fig. 13 for all four designable parameters. The progress of the optimization is summarized in Table IV. It is evident that the direct-Broyden derivatives do not converge well toward the reference values, and the objective function converges to a different (worse) solution than that of the 
TABLE IV

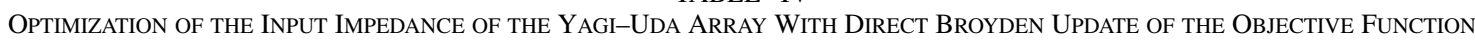

\begin{tabular}{cccccccc}
\hline$k$ & $l_{1 n}$ & $l_{2 n}$ & $s_{1 n}$ & $s_{2 n}$ & $R_{\text {in }}$ & $X_{\text {in }}$ & $f$ \\
\hline 1 & 0.450000 & 0.450000 & 0.250000 & 0.250000 & 39.08 & -9.217 & 28.5796 \\
2 & 0.459375 & 0.459375 & 0.259375 & 0.259375 & 47.91 & 11.080 & 22.5489 \\
3 & 0.459366 & 0.459375 & 0.259366 & 0.259366 & 47.91 & 11.049 & 22.4900 \\
4 & 0.449406 & 0.459245 & 0.257112 & 0.268156 & 43.81 & -3.544 & 14.2631 \\
5 & 0.449400 & 0.459245 & 0.257093 & 0.268172 & 43.81 & -3.517 & 14.2381 \\
6 & 0.453993 & 0.459195 & 0.256243 & 0.272291 & 45.26 & 4.200 & 12.6521 \\
7 & 0.453994 & 0.459195 & 0.256243 & 0.272291 & 45.26 & 4.200 & 12.6521 \\
8 & 0.453993 & 0.459195 & 0.256243 & 0.272291 & 45.26 & 4.200 & 12.6519 \\
\hline
\end{tabular}

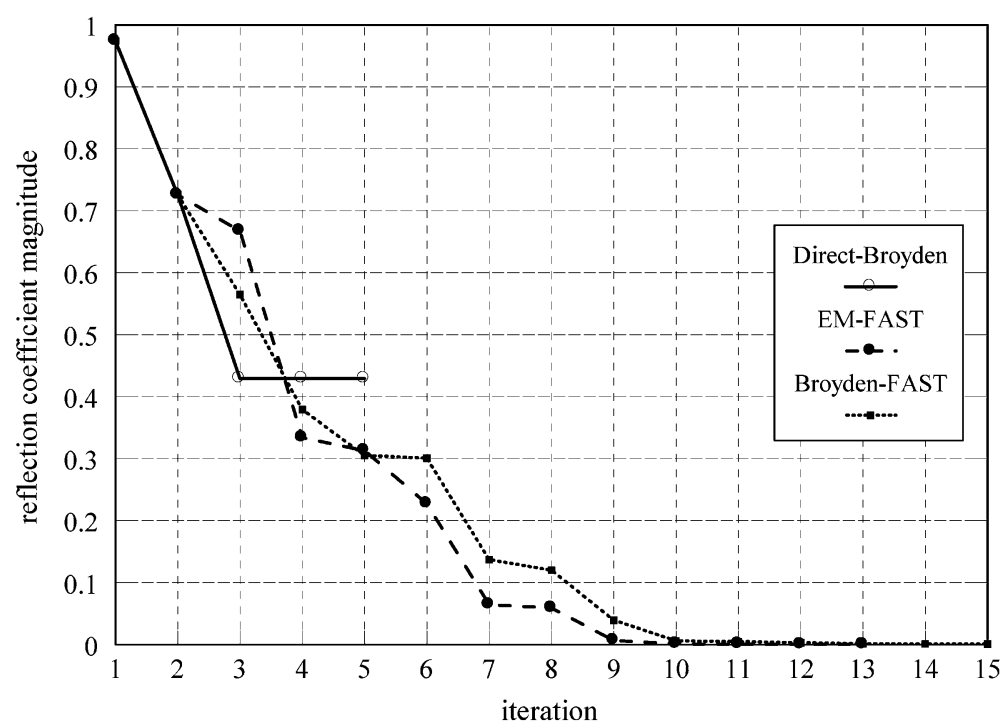

Fig. 14. Progress of the objective function during the optimization of the microstrip-fed patch antenna.

Broyden-FAST optimization. This is due mostly to three interrelated factors: (1) the objective function is very sensitive to the designable parameters (especially $l_{2 n}$ and $s_{1 n}$ ); (2) the Broyden sensitivity estimation can not track well such a rapidly changing function; (3) the incorrect sensitivity information misleads the optimizer. Possible solutions to the problems encountered with the direct Broyden sensitivity analysis are provided by the trust region optimization approaches. This topic, however, is outside of the scope of our work. With this example, we only illustrate the improved convergence of both the sensitivity analysis and the optimization when the Broyden update is applied at the level of the system matrix.

2) Optimization of a Microstrip-Fed Patch Antenna: We now apply the Broyden-FAST to the optimization of the patch antenna in Fig. 4. The length $L$ and the width $W$ of the patch are optimized for a minimum magnitude of the reflection coefficient. Thus, the objective function to be minimized is

$$
f(\boldsymbol{x})=\left|\frac{Z_{\text {in }}-Z_{0}}{Z_{\text {in }}+Z_{0}}\right|, \quad x=\left[\begin{array}{ll}
L & W
\end{array}\right]^{T} .
$$

Here, $Z_{\text {in }}$ is the input impedance of the antenna, and $Z_{0}$ is the characteristic impedance of the feeding microstrip line, which approximately equals $50 \Omega$ in this example. The operating frequency is $2 \mathrm{GHz}$. The initial values of the designable parameters are $L=55 \mathrm{~mm}$ and $W=85 \mathrm{~mm}$. The patch is meshed with rectangular segments. The number of segments along the length and the width of the patch are 11 and 17, respectively. One segment is used along the width of the feeding microstrip line. The optimization is carried out using the fmincon function of MATLAB.

We compute the sensitivities of the objective function with three methods: 1) the direct-Broyden update; 2) the traditional EM-FAST which employs finite differences to approximate the derivative matrices (without a boundary layer); and 3) the proposed Broyden-FAST which employs the Broyden update at the level of the system matrix. This time, we run three optimizations, each being driven by the respective sensitivity analysis technique. The three objective functions are plotted in Fig. 14 versus the optimization iteration number. The design parameters versus the iteration number are plotted in Fig. 15. It is clear from both figures that the direct-Broyden method fails in meeting the optimum design while the other two methods, EM-FAST and Broyden-FAST, are capable of achieving the optimum design. The obtained optimal patch dimensions are $L^{*}=46.43 \mathrm{~mm}$ and $W^{*}=99.78 \mathrm{~mm}$. 


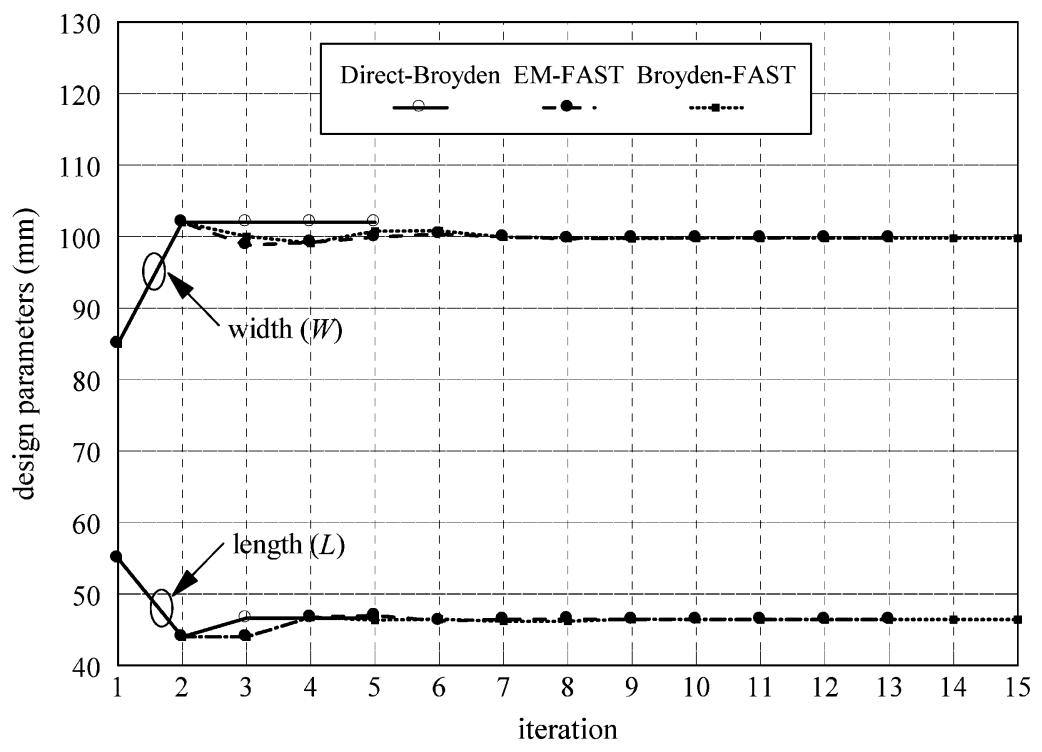

Fig. 15. Progress of the design parameters during the optimization of the microstrip-fed patch antenna.

The reason that Broyden-FAST succeeded where the directBroyden approach failed is similar to the reason outlined in the previous example with the Yagi-Uda antenna. The directBroyden approach is incapable to represent properly the fast variations in the sensitivities especially close to an optimal solution, which is associated with a resonance. On the other hand, Broyden-FAST deals with the sensitivities of the $\boldsymbol{Z}$-matrix elements, which are slowly varying functions unaffected by resonance.

\section{CONCLUSION}

We propose a novel technique for accelerated gradient based optimization. It integrates the recently developed feasible adjoint sensitivity technique for full-wave EM analysis [17] with methods for accelerated estimation of the derivatives of the system matrix. We consider two such methods: the boundary-layer concept and the Broyden update, and investigate their accuracy and versatility. We show that the Broyden update of the matrix derivatives is efficient, problem-independent and sufficiently accurate for the purpose of gradient based optimization. When integrated with our feasible adjoint sensitivity technique, it allows the computation of the system response and its gradient in the design parameter space through a single system analysis. The overhead associated with the gradient estimation is negligible in comparison with the computational requirements of the full-wave analysis. Our Broyden-FAST technique combines the efficiency of the adjoint-variable sensitivity analysis with the simplicity of the Broyden update. Its implementation is straightforward and does not require any analytical preprocessing.

\section{REFERENCES}

[1] J. W. Bandler, Q.-J. Zhang, J. Song, and R. M. Biernacki, "FAST gradient based yield optimization of nonlinear circuits," IEEE Trans. Microwave Theory Tech., vol. 38, pp. 1701-1710, Nov. 1990.

[2] J. Ureel and D. De Zutter, "A new method for obtaining the shape sensitivities of planar microstrip structures by a full-wave analysis," IEEE Trans. Microwave Theory Tech., vol. 44, pp. 249-260, Feb. 1996.
[3] E. J. Haug, K. K. Choi, and V. Komkov, Design Sensitivity Analysis of Structural Systems. Orlando, FL: Academic, 1986.

[4] A. D. Belegundu and T. R. Chandrupatla, Optimization Concepts and Applications in Engineering. Upper Saddle River, NJ: Prentice Hall, 1999.

[5] R. Tomoviæ, Sensitivity Analysis of Dynamic Systems. New York: McGraw-Hill, 1963.

[6] J. W. Bandler, "Computer-aided circuit optimization," in Modern Filter Theory and Design, G. C. Temes and S. K. Mitra, Eds. New York: Wiley, 1973, ch. 6.

[7] K. C. Gupta, R. Garg, and R. Chadha, Computer-Aided Design of Microwave Circuits. Dedham, MA: Artech, 1981.

[8] J. W. Bandler and R. E. Seviora, "Current trends in network optimization," IEEE Trans. Microwave Theory Tech., vol. MTT-18, pp. 1159-1170, Dec. 1970.

[9] - "Wave sensitivities of networks," IEEE Trans. Microwave Theory Tech., vol. MTT-20, pp. 138-147, Feb. 1972.

[10] G. Iuculano, V. A. Monaco, and P. Tiberio, "Network sensitivities in terms of scattering parameters," Electron. Lett., vol. 7, pp. 53-55, Jan. 1971.

[11] J. W. Bandler, Q. J. Zhang, and R. M. Biernacki, "A unified theory for frequency-domain simulation and sensitivity analysis of linear and nonlinear circuits," IEEE Trans. Microwave Theory Tech., vol. AP-36, pp. 1661-1669, Dec. 1988.

[12] F. Alessandri, M. Mongiardo, and R. Sorrentino, "New efficient full wave optimization of microwave circuits by the adjoint network method," IEEE Microwave and Guided Wave Letters, vol. 3, pp. 414-416, Nov. 1993.

[13] H. Akel and J. P. Webb, "Design sensitivities for scattering-matrix calculation with tetrahedral edge elements," IEEE Trans. Magn., vol. 36, pp. 1043-1046, July 2000.

[14] H.-B. Lee and T. Itoh, "A systematic optimum design of waveguide-tomicrostrip transition," IEEE Trans. Microwave Theory Tech., vol. 45, pp. 803-809, May 1997.

[15] J. P. Webb, "Design sensitivity of frequency response in 3-D finite-element analysis of microwave devices," IEEE Trans. Magnetics, vol. 38, pp. 1109-1112, Mar. 2002.

[16] J. Ureel and D. De Zutter, "Shape sensitivities of capacitances of planar conducting surfaces using the method of moments," IEEE Trans. Microwave Theory Tech., vol. 44, pp. 198-207, Feb. 1996.

[17] N. K. Georgieva, S. Glavic, M. H. Bakr, and J. W. Bandler, "Feasible adjoint sensitivity technique for EM design optimization," IEEE Trans. Microwave Theory Tech., vol. 50, pp. 51-2758, Dec. 2002.

[18] N. K. Nikolova, J. W. Bandler, and M. H. Bakr, "Adjoint techniques for sensitivity analysis in high-frequency structure CAD," IEEE Trans. Microwave Theory Tech., Special Issue on Electromagnetics-Based Optimization of Microwave Components and Circuits, to be published.

[19] S. Amari, "Numerical cost of gradient computation within the method of moments and its reduction by means of a novel boundary-layer concept," in Proc. IEEE MTT-S Int. Symp. Dig., vol. 3, 2001, pp. 1945-1948. 
[20] C. A. Balanis, Antenna Theory, 2nd ed. New York: Wiley, 1997.

[21] C. G. Broyden, "A class of methods for solving nonlinear simultaneous equations," Mathematics of Computation, vol. 19, pp. 577-593, 1965.

[22] J. W. Bandler, S. H. Chen, S. Daijavad, and K. Madsen, "Efficient optimization with integrated gradient approximations," IEEE Trans. Microwave Theory Tech., vol. 36, pp. 444-455, Feb. 1988.

[23] J. W. Bandler, R. M. Biernacki, S. H. Chen, R. H. Hemmers, and K. Madsen, "Electromagnetic optimization exploiting aggressive space mapping," IEEE Trans. Microwave Theory Tech., vol. 43, pp. 2874-2882, Dec. 1995

[24] S. Glavic, "Electromagnetic Design Sensitivity Analysis of High-Frequency Structures," M.A.Sc. thesis, McMaster University, May 2002.

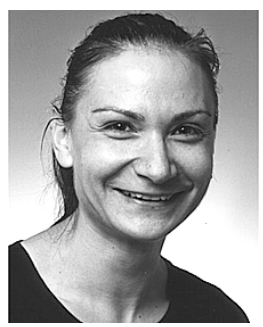

Natalia K. Nikolova (formerly Georgieva) (S'93-M'97) received the Dipl.Eng. degree in radioelectronics from the Technical University of Varna, Bulgaria, in 1989 and the Ph.D. degree in electrical engineering from the University of Electro-Communications, Tokyo, Japan, in 1997.

From 1998 to 1999, she held a Postdoctoral Fellowship of the Natural Sciences and Engineering Research Council of Canada (NSERC), during which time she was initially with the Microwave and Electromagnetics Laboratory, DalTech, Dalhousie University, Halifax, Canada. For approximately one year, she was with the Simulation Optimization Systems Research Laboratory, McMaster University, Hamilton, ON, Canada. In July 1999, she joined the Department of Electrical and Computer Engineering, McMaster University, where she is currently an Associate Professor. Her research interests include theoretical and computational electromagnetism, high-frequency analysis techniques, as well as CAD methods for high-frequency structures and antennas.

Dr. Nikolova currently holds a University Faculty Award of NSERC, which she received in 2000

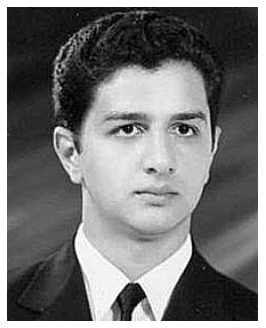

Reza Safian received the B.Sc. degree from the Isfahan University of Technology (IUT), Isfahan, Iran, in 1999 and the M.A.Sc. degree from McMaster University, Hamilton, Canada, in 2003, both in electrical engineering. He is currently working toward the Ph.D. degree at the University of Toronto, Toronto, ON, Canada.

During 1999 to 2002, he was a Research Engineer in the Electrical and Computer Engineering Research Center (ECERC), IUT. From 2002 to 2003, he was a Research Assistant at McMaster University working with the Computational Electromagnetics Laboratory and the Simulation Optimization Systems Research Laboratory. His research interests include theory of electromagnetism and computational electromagnetics.

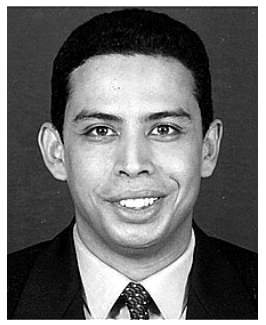

Ezzeldin A. Soliman (S'97-A'99) was born in Cairo, Egypt, on May 18, 1970. He received the B.Sc. degree (distinction with honors) in electronics and communications engineering and the M.Sc. degree in engineering physics, both from Cairo University, Giza, Egypt, in June 1992 and Nov. 1995, respectively, and the Ph.D. degree (summa cum laude) in electrical engineering from the University of Leuven, Leuven, Belgium, in February 2000.

From 1992 to 1996, he was a Research and a Teaching Assistant with the Department of Engineering Physics, Faculty of Engineering, Cairo University. From 1996 to 2000, he has been a Research Assistant at both Interuniversity MicroElectronics Center (IMEC), Leuven, Belgium, and the Department of Electrical Engineering, University of Leuven. From April 2002 to July 2002, he was a Visiting Professor at IMEC. From October 2002 to September 2003, he was on a Visiting Researcher in the Department of Electrical and Computer Engineering, McMaster University, Hamilton, ON, Canada. He is currently an Assistant Professor with the Department of Engineering Physics, Faculty of Engineering, Cairo University. His research interests include computational electromagnetics, development and characterization of planar antennas in the multilayer thin film technology, neural network modeling of electromagnetic problems, and the EM-based optimization techniques.

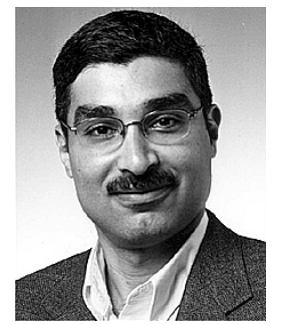

Mohamed H. Bakr (S'98-M'00) received the B.Sc degree in electronics and communications engineering (distinction with honors) and the Master's degree in engineering mathematics from Cairo University, Giza, Egypt, in 1992 and 1996, respectively, and the Ph.D. degree from the Department of Electrical and Computer Engineering, McMaster University, Hamilton, ON, Canada, in September 2000.

In 1997, he was a Student Intern with Optimization Systems Associates (OSA), Inc. From 1998 to 2000, he worked as a Research Assistant with the Simulation Optimization Systems (SOS) Research Laboratory, McMaster University. In November 2000, he joined the Computational Electromagnetics Research Laboratory (CERL), University of Victoria, Victoria, Canada as an NSERC Postdoctoral Fellow. He is currently an Assistant Professor with the Department of Electrical and Computer Engineering, McMaster University. His research areas of interest include optimization methods, computer-aided design and modeling of microwave circuits, neural network applications, smart analysis of microwave circuits and efficient optimization using time/frequency domain methods.

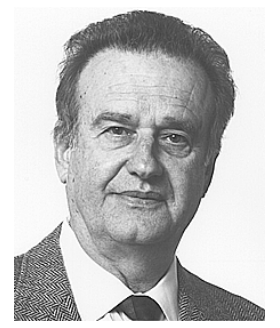

John W. Bandler (S'66-M'66-SM'74-F'78) was born in Jerusalem, on November 9, 1941. He studied at the Imperial College of Science and Technology, London, U.K., from 1960 to 1966, and received the B.Sc.(Eng.), Ph.D., and D.Sc.(Eng.) degrees from the University of London, London, U.K., in 1963, 1967, and 1976, respectively.

In 1966, he joined Mullard Research Laboratories, Redhill, Surrey, U.K. From 1967 to 1969, he was a Postdoctorate Fellow and Sessional Lecturer at the University of Manitoba, Winnipeg, Canada. He joined McMaster University, Hamilton, ON, Canada, in 1969, where he has served as Chairman of the Department of Electrical Engineering and Dean of the Faculty of Engineering, and is currently Professor Emeritus in the Electrical and Computer Engineering Department, directing research in the Simulation Optimization Systems Research Laboratory. He was President of Optimization Systems Associates Inc. (OSA), which he founded in 1983, until November 20, 1997, the date of acquisition of OSA by Hewlett-Packard Company (HP). OSA implemented a first-generation yield-driven microwave CAD capability for Raytheon in 1985, followed by further innovations in linear and nonlinear microwave CAD technology for the Raytheon/Texas Instruments Joint Venture MIMIC Program. OSA introduced the CAE systems RoMPE in 1988, HarPE in 1989, OSA90 and OSA90/hope in 1991, Empipe in 1992, Empipe3D and EmpipeExpress in 1996. OSA created the product empath in 1996 which was marketed by Sonnet Software, Inc., USA. Dr. Bandler is President of Bandler Corporation, which he founded in 1997. He has published more than 350 papers from 1965 to 2003. He contributed to Modern Filter Theory and Design (Surrey, U.K.: Wiley-Interscience, 1973) an to Analog Methods for Computer-aided Analysis and Diagnosis Germany: Marcel Dekker, Inc., 1988). Four of his papers have been reprinted in Computer-Aided Filter Design (New York: IEEE Press, 1973), one in each of Microwave Integrated Circuits (Norwood, MA: Artech House, 1975), Low-Noise Microwave Transistors and Amplifiers (New York: IEEE Press, 1981), Microwave Integrated Circuits, (Norwood, MA: Artech House, 1985, 2nd ed.), Statistical Design of Integrated Circuits (New York: IEEE Press, 1987)and Analog Fault Diagnosis (New York: IEEE Press, 1987). He joined the Editorial Boards of the International Journal of Numerical Modeling in 1987, the International Journal of Microwave and Millimeterwave Computer-Aided Engineering in 1989, and Optimization and Engineering in 1998 He was Guest Editor of International Journal of Microwave and Millimeter-Wave Computer-Aided Engineering, Special Issue on Optimization-Oriented Microwave CAD (1997). He was Guest Coeditor Optimization and Engineering Special Issue on Surrogate Modeling and Space Mapping for Engineering Optimization (2001).

Dr. Bandler is a Fellow of the Canadian Academy of Engineering, the Royal Society of Canada, the Institution of Electrical Engineers (IEE), London, U.K., anf the Engineering Institute of Canada. He is a Member of the Association of Professional Engineers of the Province of Ontario (Canada), the MIT Electromagnetics Academy, and the Micronet Network of Centres of Excellence. He received the Automatic Radio Frequency Techniques Group (ARFTG) Automated Measurements Career Award in 1994. He was an Associate Editor of the IEEE TRANSACTIONS ON MiCROWAVE THEORY AND TECHNiQUeS (1969-1974), and has continued serving as a member of the Editorial Board. He was Guest Editor of the IEEE TRANSACTIONS ON MicrowaVE THEORY AND TeChNioues Special Issue on computer-oriented microwave practices (1974) and Guest Coeditor of the of the IEEE TRANSACTIONS ON MicrowaVe THEORY AND TeChNiQues Special Issue on Process-Oriented Microwave CAD and Modeling (1992), and Guest Editor, IEEE TRANSACtions on Microwave Theory and TeChNioues, Special Issue on Automated Circuit Design Using Electromagnetic Simulators (1997). He is Guest Coeditor, IEEE TRANSACTIONS ON Microwave TheORY AND TeChNiQues, Specia Issue on Electromagnetics-Based Optimization of Microwave Components and Circuits (2004). He has served as Chair of the MTT-1 Technical Committee on Computer-Aided Design. 